\title{
Time-resolved EPR investigation of oxygen and temperature effects on synthetic eumelanin
}

\author{
Fosca Conti $^{\mathrm{a}, *}$, Lucia Panzella ${ }^{\mathrm{b}}$, Alessandra Napolitano ${ }^{\mathrm{b}}$, Marco d'Ischia ${ }^{\mathrm{b}}$ and \\ Antonio Toffoletti ${ }^{\text {a }}$ \\ ${ }^{a}$ Department of Chemical Sciences, University of Padova, Padova, Italy \\ ${ }^{\mathrm{b}}$ Department of Organic Chemistry and Biochemistry, University of Naples Federico II, Naples, Italy
}

\begin{abstract}
Synthetic eumelanin produced using 5,6-dihydroxyindole-2-carboxylic acid as precursor and $\mathrm{H}_{2} \mathrm{O}_{2}$ /horseradish peroxidase as oxidative reagent, in form of dry powder, has been investigated under photoexcitation by TR-EPR spectroscopy. The formation of spin polarized radical pairs from triplet excited states of melanin has been observed both in absence and in presence of oxygen and has been followed as a function of the temperature in the range 140-290 K. The triplet mechanism explains the observed polarization pattern in net emission. In the presence of oxygen new radical pairs are formed by interaction of melanin with molecular oxygen.
\end{abstract}

Keywords: Melanin, DHICA, EPR spectroscopy, radical pair, spin polarization, excited state, superoxide radical anion

\section{Introduction}

Eumelanins are biopolymers which play a key role in the mammalian pigmentation system. In humans they are present in skin, hair and eyes and are responsible for the dark colours, in contrast to pheomelanins which are responsible of the red-yellow pigmentation. Eumelanins derive biogenetically from tyrosine via the oxidative polymerization of 5,6-dihydroxyindoles [15].

Although studied extensively in the last decades, the eumelanin role has not been fully elucidated. One effective strategy for investigation is the use of Electron Paramagnetic Resonance (EPR) spectroscopic techniques because radical species are found in natural and synthetic eumelanins. The EPR signal is permanent and consists of a single, slightly asymmetric line with a peak-to-peak width of $0.4-0.6 \mathrm{mT}$ and a $g$-factor close to 2.004. It has been attributed to intrinsic semiquinone like radicals involved in redox equilibrium between quinone and hydroquinone units [1,11,14,20,21]. Under UV and visible irradiation the line intensity changes and a slight shift of the $g$-factor is observable, indicating the formation of new light-generated species (extrinsic radicals). By using Time-Resolved EPR (TR-EPR) spectroscopy, Norris and co-workers proposed that in the photoproduction of new melanin free radicals, they are generated as radical pairs (RPs) from the triplet manifold. The RPs were observed in retinal pigment epithelium (RPE) cells of human eyes [18,19], and in Sepia [17] and porcine [23] eumelanin. Recently,

\footnotetext{
${ }^{*}$ Corresponding author: Dr. Fosca Conti, Department of Chemical Sciences, University of Padova, via Marzolo 1, I-35131 Padova, Italy. E-mail: fosca.conti@ unipd.it.
} 
we have reported on the combined effect of molecular oxygen and photoexcitation on the generation of radicals in a synthetic eumelanin produced using 5,6-dihydroxyindole (DHI) as precursor [22]. In the presence of oxygen, we observed TR-EPR spectra with a polarization pattern along the magnetic field typical of RPs in which the electron spin polarization is produced by the radical pair mechanism $[8,10]$. To explain our results we proposed that RPs are formed by interaction of melanin with $\mathrm{O}_{2}$.

In this paper we report on TR-EPR investigation on a synthetic eumelanin produced using 5,6dihydroxyindole-2-carboxylic acid (DHICA) as precursor. Our main motivation is to understand the functionality of melanin in presence of oxygen since it is present in living cells. We compare the spectroscopic results with those observed for synthetic eumelanin produced using DHI as precursor and the same oxidant reagent [22]. We consider the process involving molecular oxygen and the time evolution of the electron spin polarization as a function of temperature in the range 140-290 K.

\section{Experimental}

\subsection{Synthesis of eumelanin 1 and sample preparation for EPR measurements}

Eumelanin 1 was produced using 5,6-dihydroxyindole-2-carboxylic acid (DHICA) as precursor and $\mathrm{H}_{2} \mathrm{O}_{2} / \mathrm{HRP}$ (horseradish peroxidase) as oxidative reagent [12]. Briefly, $\mathrm{HRP}\left(16 \mathrm{U} \mathrm{ml}^{-1}\right)$ and $30 \% \mathrm{H}_{2} \mathrm{O}_{2}$ $(124 \mu \mathrm{l})$ were added to DHICA $(200 \mathrm{mg}, 1.04 \mathrm{mmol})$ dissolved in $0.1 \mathrm{M}$ phosphate buffer, $\mathrm{pH} 7.4$ $(83 \mathrm{ml})$, and the mixture was taken under vigorous stirring at room temperature for $2 \mathrm{~h}$. After careful acidification to $\mathrm{pH} 3$ with $2 \mathrm{M} \mathrm{HCl}$, the pigment which separated was collected by centrifugation at $6000 \mathrm{rpm}$ for $30 \mathrm{~min}$ at $4{ }^{\circ} \mathrm{C}$, washed five times with water and lyophilized $(125 \mathrm{mg}$ ).

For TR-EPR experiments we have prepared two types of samples: ca. $5 \mathrm{mg}$ of dry eumelanin 1 in powder, i.e. without any solvent, in the absence of oxygen and the same amount of eumelanin 1 in powder in equilibrium with oxygen of the air. For the sample without oxygen, the EPR tube containing 1 was connected to a vacuum line, the powder was degassed by repeated freeze-pump-thaw cycles and sealed under vacuum $\left(10^{-5}\right.$ Torr). Even if the melanin powder has been lyophilized before the preparation of the EPR samples, a small and variable amount of water remains in the solid. This is witnessed by the reduction of the quality factor of the spectrometer microwave cavity observed for some of the melanin samples. This problem has been overcome using EPR tubes with a small diameter ( $2 \mathrm{~mm}$ i.d.).

\subsection{TR-EPR instrumentation}

The experimental setup used for TR-EPR measurements comprises a conventional X-band EPR spectrometer (Bruker ER 200 D) equipped with a nitrogen flow cryostat for temperature control. For optical excitation, the second harmonic $(\lambda=532 \mathrm{~nm})$ of a Nd/YAG pulsed solid-state laser (Brilliant Quantel) was used ( $5 \mathrm{~ns}$ pulse width, $20 \mathrm{~Hz}$ repetition rate and $5 \mathrm{~mJ}$ energy per pulse). The EPR transient signals, generated by the laser pulses, were recorded without field modulation, with a preamplifier (bandwidth: $20 \mathrm{~Hz}-6.5 \mathrm{MHz}$ ) and a digital oscilloscope (LeCroy LT344) that averages them. A two-dimensional surface where the EPR signal is recorded vs. time and magnetic field B (2D TR-EPR spectrum) is obtained by sweeping B in a selected interval and collecting EPR transient signals at each field position. To correct the EPR data for the cavity response to the laser perturbation, an off-resonance signal is subtracted from the averaged transient signals recorded at all the field positions of the 2D TR-EPR surface. Since in the spectra the EPR signal was recorded without magnetic field modulation (direct detection mode), positive or negative peaks represent transitions in enhanced absorption (A) or in emission (E), respectively. 


\section{Results and discussion}

One of the limiting factor in the study of eumelanins is their insolubility in most solvents. For this reason our investigation was carried out on solid samples. The TR-EPR spectrum of eumelanin 1 as dry powder, in vacuum and under laser excitation shows a strongly spin polarized signal completely in emission in the temperature range 140-290 K. Spin polarized signals are observed when the populations of the spin energy levels differ from those at thermal equilibrium (Boltzmann populations). This phenomenon produces strongly enhanced EPR signal, either in enhanced absorption (A) or even in emission (E), as in the present case. Figure 1a shows the complete 2D TR-EPR surface at $290 \mathrm{~K}$. The emissive line is centered at $g=2.0047 \pm 0.0005$ and has Gaussian shape with $0.7 \mathrm{mT}$ linewidth and a slight asymmetry due to $g$ anisotropy (see Fig. 1b, showing the spectrum at the time delay corresponding to the maximum of the transient signal). The time evolution is a mono-exponential decay with a time constant of $(13.3 \pm 0.5) \mu$ s (Fig. 1c). These features are comparable to those observed for natural melanin in RPE cells [17-19,23] and synthetic melanin produced starting from DHI as precursor [22]. Similarly, the emissive signal can be attributed to the radical pairs generated from the excited triplet manifold of melanin $[4,5]$. This result provides a strong evidence which supports the existence of excited triplet states in melanin, in contrast with the general held views [11]. The intersystem crossing to excited triplet states
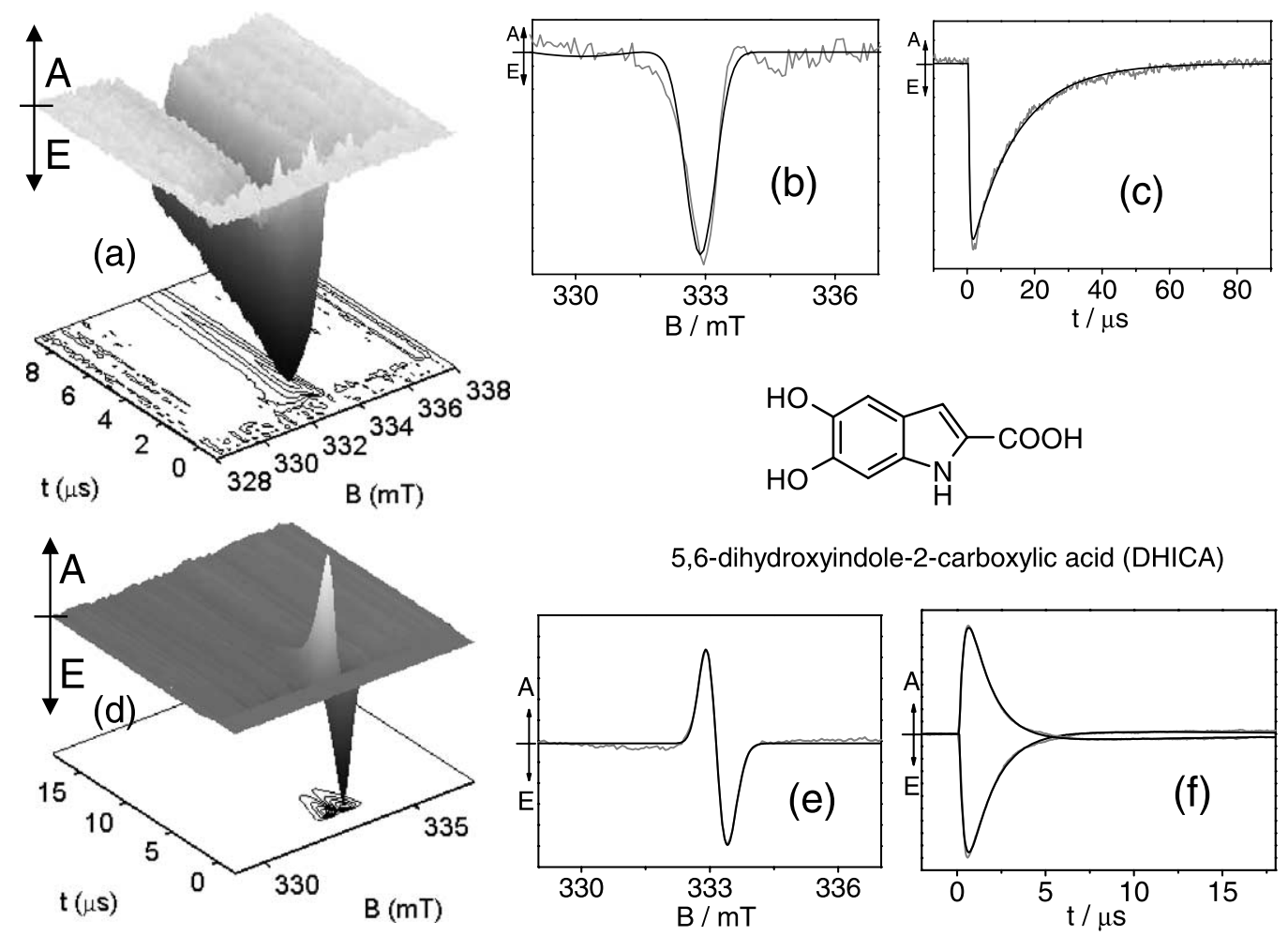

Fig. 1. TR-EPR signal of eumelanin 1 dry powder at $290 \mathrm{~K}$ : (a) complete 2D spectrum of 1 under vacuum; (b) spectrum at a delay of $2 \mu \mathrm{s}$ after the laser pulse (maximum of the transient signal) (gray) and Gaussian fitting (black); (c) transient EPR signal at $B=332.9 \mathrm{mT}$ (maximum of emission) (gray) and mono-exponential fitting (black); (d) complete 2D spectrum of 1 in equilibrium with oxygen in the air; (e) spectrum at a delay of $0.5 \mu$ s after the laser pulse (maximum of the transient signal) (gray) and Gaussian fitting (black); (f) transient EPR signals (gray) and bi-exponential fittings (black) at the maximum of absorption and at the maximum of emission (A - enhanced absorption, $\mathrm{E}$ - emission). 

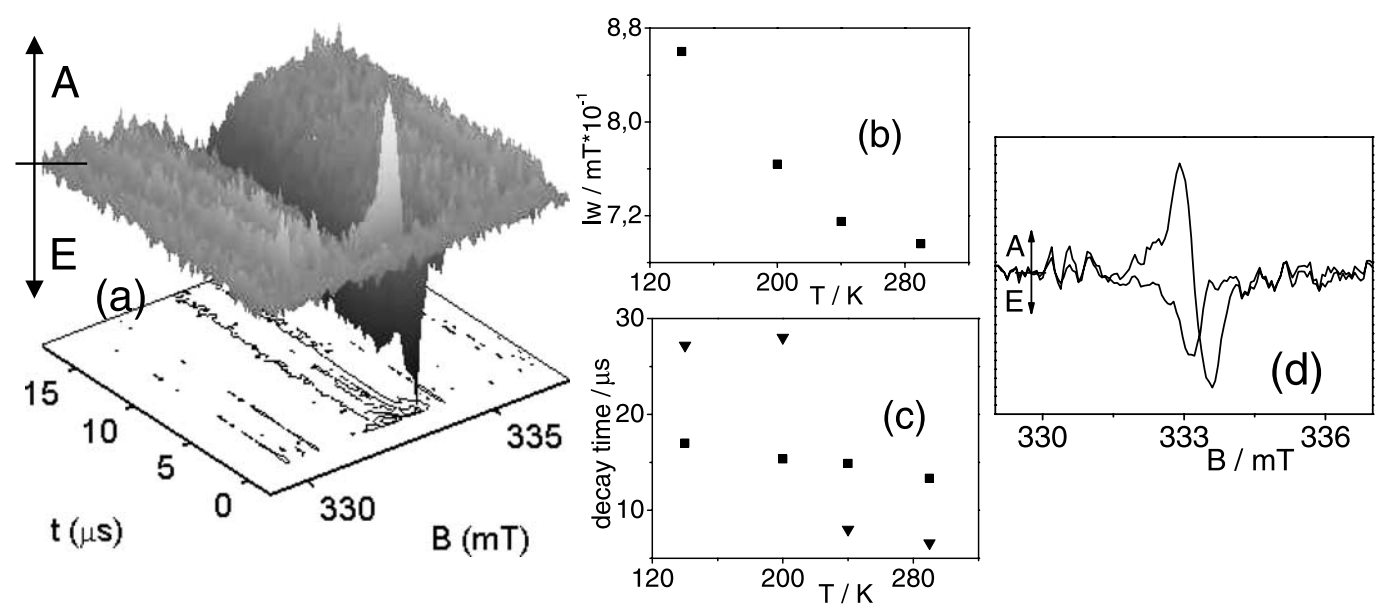

Fig. 2. (a) Complete 2D TR-EPR spectrum of eumelanin 1 dry powder in equilibrium with oxygen in the air at $140 \mathrm{~K}$; (b) temperature dependence of the Gaussian linewidth (lw) of the EPR spectrum of 1 under vacuum recorded $2 \mu$ s after the laser pulse; (c) temperature dependence of the monoexponential decay time of the emissive EPR signal of eumelanin 1 under vacuum (squares) and in equilibrium with oxygen in the air (triangles); (d) sections of the 2D TR-EPR spectrum of Fig. 2a extracted at a delay, after the laser pulse, of $0.5 \mu$ s (A/E pattern) and $2 \mu$ s (E pattern).

is fast enough to not be precluded by the quick conversion of light energy into heat and photochemical reactions. We propose that the polarization pattern in net emission is due to the triplet mechanism, which gives rise to EPR spectra in which all lines have the same polarization [8,24].

In order to characterize the emissive peak, the TR-EPR signals were recorded as a function of the temperature. No additional features are present in the spectra in the range 140-290 K. An increase of ca. $20 \%$ in linewidth is observed with decreasing temperature (Fig. 2b) for eumelanin samples in EPR tube sealed under vacuum. This feature can be explained in terms of mobility of the melaninmelanin radical pairs. The contribution of anisotropic magnetic interactions (i.e., $g$ and hyperfine tensors) to the linewidth at high temperature can be partially averaged out by molecular motion even in solid samples. Additionally, the decay time of the emissive trace decreases by ca. $25 \%$ from 140 to $290 \mathrm{~K}$, as indicated by squares in Fig. 2c. As for the maximum amplitude of the TR-EPR signal, by raising temperature we observe an increase of the signal which depends on several factors: the efficiency of triplet generation (ISC), the yield of radical pairs (steps 2 and 3 in the mechanism proposed hereinafter), the rate of spin relaxation, and the radical lifetimes. Therefore, we do not attempt to disentangle the different temperature dependencies of the above indicated factors.

The sample containing eumelanin 1 in an open quartz tube, i.e. in equilibrium with oxygen in the air, presents a more complex TR-EPR spectrum since two distinct signals emerge: one rising and falling within the first microseconds and a second one emerging thereafter, both with polarization pattern indicative of radical pairs. Figure 1d shows the complete 2D TR-EPR surface at $290 \mathrm{~K}$.

At earlier times after the exciting laser pulse (time delay from the pulse $<2 \mu \mathrm{s}$ ), the spectrum along the magnetic field shows two components, centered at $g=2.0043 \pm 0.0005$, the first in enhanced absorption and the second in emission (A/E pattern) (Fig. 1e). The two peaks have Gaussian shape, with 0.42 (A) and $0.53 \mathrm{mT}$ (E) linewidth respectively. The net emissive signal recorded in absence of oxygen is present, even if barely observable in Fig. 1d, and it has to be considered in the simulations of the time traces of the EPR signal at the field position corresponding to the maximum of absorption and maximum of emission reported in Fig. 1f. The transients are fitted using a bi-exponential decay with 
time constants $1.30 \pm 0.05$ and $15.2 \pm 0.5 \mu$ s, which includes the contribution of the two distinct signals. The long time constant is comparable with that observed for the sample in absence of oxygen. By decreasing the temperature and after prolonged laser excitation the intensity of the slow emissive signal increases in comparison with the A/E signal intensity, as reported in the TR-EPR surface recorded at $140 \mathrm{~K}$ (Fig. 2a). It is clear that the mechanism of photoproduction of new radical pairs in melanin is affected by molecular oxygen. At this point two more comments are necessary. (1) Different samples of melanin powder containing variable amount of residual water have been used for TR-EPR experiments, but no change in the polarization pattern has been noticed. Moreover, preliminary experiments with melanin suspended in water have given similar results, i.e. all emissive signal (E) in the absence of oxygen and $\mathrm{A} / \mathrm{E}$ followed by $\mathrm{E}$ signals in presence of oxygen. This indicates that water does not play a substantial role in the photoproduction of radical pairs. (2) The presence of water in melanin could in principle affect slightly the hyperfine interactions in the radicals. Anyway in our spectra the hyperfine structure is not resolved (both in air and in vacuum) and no effect on the linewidth has been observed.

We suggest the following mechanisms for the creation of two different RPs:

$$
\begin{aligned}
& { }^{1} \mathrm{M}_{i} \stackrel{h \nu}{\longrightarrow}{ }^{1}\left(\mathrm{M}_{i}\right)^{*} \\
& { }^{1}\left(\mathrm{M}_{i}\right)^{*} \longrightarrow{ }^{3}\left(\mathrm{M}_{i}\right)^{*} \\
& { }^{3}\left(\mathrm{M}_{i}\right)^{*}+{ }^{1} \mathrm{M}_{j} \longrightarrow\left[{ }^{2} \mathrm{M}_{i} \cdot+\ldots{ }^{2} \mathrm{M}_{j}{ }^{\bullet-}\right] \\
& { }^{1}\left(\mathrm{M}_{i}\right)^{*}+{ }^{3} \mathrm{O}_{2} \longrightarrow{ }^{3}\left(\mathrm{M}_{i}\right)^{*}+{ }^{1} \mathrm{O}_{2}{ }^{*} \\
& { }^{1} \mathrm{O}_{2}{ }^{*}+{ }^{1} \mathrm{M}_{k} \mathrm{H}^{-} \rightarrow{ }^{1}\left[{ }^{1} \mathrm{O}_{2}{ }^{*} \ldots{ }^{1} \mathrm{M}_{k} \mathrm{H}^{-}\right] \rightarrow{ }^{1}\left[{ }^{2} \mathrm{O}_{2} \cdot-\ldots{ }^{2} \mathrm{MH}_{k}{ }^{\bullet}\right]
\end{aligned}
$$

After photoexcitation to the excited singlet state, step (1), melanin ${ }^{1} \mathrm{M}^{*}$ can either convert via intersystem crossing (ISC) process to the excited triplet state ${ }^{3} \mathrm{M}^{*}$, step (2), or interact with molecular oxygen, step (4). The ISC process explains the formation of RPs which gives the long lasting EPR signal (all in emission) in the sample under vacuum (Fig. 1a).

In presence of oxygen, an energy transfer process can take place and excited singlet oxygen ${ }^{1} \mathrm{O}_{2}{ }^{*}$ can be formed [16]. The electron transfer reaction in step (5) accounts for the radical pair generation giving the A/E EPR signal at short delays from the laser pulse. Of course, after steps (3) and (5) the two radicals can eventually separate and/or undergo further reactions.

Following the discussion reported in our previous work on synthetic melanin produced by using DHI as precursor [22], we attribute the $\mathrm{A} / \mathrm{E}$ pattern to a radical pair in which the electron spin polarization is produced by the radical pair mechanism [8,10]: an EPR spectrum composed by two doublets of lines, centered at the two $g$ values of the partners of the pair, is expected. The polarization pattern appears $\mathrm{A} / \mathrm{E}-\mathrm{A} / \mathrm{E}$ or $\mathrm{E} / \mathrm{A}-\mathrm{E} / \mathrm{A}$ in relation to the sign of the exchange and dipolar interactions. In our TR-EPR spectrum only one doublet with A/E pattern is present (see Fig. 1e). The involved superoxide radical $\mathrm{O}_{2}{ }^{\bullet-}$ has axial $g$-tensor with large anisotropy $\left(g_{\|}=2.081\right.$ and $\left.g_{\perp}=2.001\right)$ [9]. When one radical has a large $g$-factor anisotropy one of the doublet-like signals is spread over a large magnetic field range and produces a very small EPR signal intensity, which is not visible anymore. Therefore, we are confident that one of the partner in RP is the superoxide radical anion. Moreover, the photogeneration of $\mathrm{O}_{2}{ }^{\bullet-}$ by natural melanins in the presence of oxygen has been already reported $[3,6,7,13]$.

Figure $2 \mathrm{c}$ shows the decay time as function of the temperature for the signal due to the RPs spin polarized in net emission. For eumelanin in equilibrium with oxygen in the air the time constant increases much going from 290 to $140 \mathrm{~K}$ (triangles) while the increment is much smaller (ca. 25\%) for the sample 
under vacuum (squares). To analyze the time evolution in presence of oxygen, the transients are extracted at the magnetic field corresponding to the zero intensity of the A/E pattern. As in the presence of molecular oxygen step (4) competes with step (2), the processes of formation of the two types of radical pairs are not independent. At room temperature, step (4) is dominant and the net emissive contribution is barely observable (Fig. 1d). Differently, at $140 \mathrm{~K}$ the two competitive processes have similar probability, producing comparable signal intensities (see Fig. 2d). On the contrary, we note that in the case of melanin synthesized from DHI [22] using the same oxidative reagent $\left(\mathrm{H}_{2} \mathrm{O}_{2} / \mathrm{HRP}\right)$, the competitive steps (2) and (4) are of comparable importance at room temperature.

\section{Conclusions}

In conclusion, we present evidence suggesting the existence of excited triplet states also for synthetic eumelanin from DHICA. Our results obtained irradiating eumelanin in the absence or in the presence of oxygen clearly indicate that the latter may play an important role in dictating eumelanin photo-behaviour. The TR-EPR experiments for eumelanin in equilibrium with oxygen in the air show that two distinct types of radical pairs are photogenerated. We propose the mechanisms of their formation. These observations are instructive for the mechanism of protection of melanin from photoinduced damage in living systems.

\section{Acknowledgement}

This research work was supported by MIUR (COFIN No. 2006034920_004).

\section{References}

[1] M. Boulton, M. Rozanowska and B. Rozanowski, J. Photochem. Photobiol. B 64 (2001), 144-161.

[2] M. d'Ischia, A. Napolitano, A. Pezzella, P. Meredith and T. Sarna, Angew. Chem. Int. Ed. Engl. 48 (2009), 3914-3921.

[3] C.C. Felix, J.S. Hyde, T. Sarna and R.C. Sealy, Biochem. Biophys. Res. Commun. 84 (1978), 335-341.

[4] C.C. Felix, J.S. Hyde and R.C. Sealy, Biochem. Biophys. Res. Commun. 88 (1979), 456-461.

[5] C.C. Felix and R.C. Sealy, Photochem. Photobiol. 34 (1981), 423-429.

[6] R.M. Haywood, M. Lee and C. Linge, J. Photochem. Photobiol. B 82 (2006), 224-235.

[7] R.M. Haywood and C. Linge, J. Photochem. Photobiol. B 76 (2004), 19-32.

[8] P.J. Hore, in: Advanced EPR. Applications in Biology and Biochemistry, A.J. Hoff, ed., Elsevier, New York, 1989, pp. 405440, Chapter 12.

[9] P.F. Knowles, J.F. Gibson, F.M. Pick and R.C. Bray, Biochem. J. 111 (1969), 53-58.

[10] G. Kothe, S. Weber, E. Ohmes, M.C. Thurnauer and J.R. Norris, J. Am. Chem. Soc. 116 (1994), 7729-7734.

[11] P. Meredith and T. Sarna, Pigment Cell Res. 19 (2006), 572-594.

[12] A. Napolitano, A. Pezzella, M.R. Vincensi and G. Prota, Tetrahedron 51 (1995), 5913-5920.

[13] M. Rozanowska, A. Bober, J. Burke and T. Sarna, Photochem. Photobiol. 65 (1997), 472-479.

[14] T. Sarna, J. Photochem. Photobiol. B 12 (1992), 215-258.

[15] T. Sarna and H.M. Swartz, in: The Pigmentary system: Its Physiology and Pathophysiology, J.J. Nordlund, R.E. Boissy, V.J. Hearing, R.A. King and J.P. Ortonne, eds, Oxford University Press, New York, 1998, pp. 311-341.

[16] R. Schmidt, Photochem. Photobiol. 82 (2006), 1161-1177.

[17] B.-L.L. Seagle, E.M. Gasyna, W.F. Mieler and J.R. Norris, Proc. Natl. Acad. Sci. USA 103 (2006), 16644-16648.

[18] B.-L.L. Seagle, K.A. Rezai, E.M. Gasyna, Y. Kobori, K.A. Rezaei and J.R. Norris, J. Am. Chem. Soc. 127 (2005), 1122011221.

[19] B.-L.L. Seagle, K.A. Rezai, Y. Kobori, E.M. Gasyna, K.A. Rezaei and J.R. Norris, Proc. Natl. Acad. Sci. USA 102 (2005), 8978-8983. 
[20] R.C. Sealy, C.C. Felix, J.S. Hyde and H.M. Swartz, in: Free Radicals in Biology, Vol. 4, W.A. Pryor, ed., Academic Press, New York, 1980, pp. 209-260.

[21] R.C. Sealy, J.S. Hyde, C.C. Felix, I.A. Menon and G. Prota, Science 217 (1982), 545-547.

[22] A. Toffoletti, F. Conti, T. Sandron, A. Napolitano, L. Panzella and M. d'Ischia, Chem. Commun. (2009), 4977-4979.

[23] A. Wang, A.R. Marino, E.M. Gasyna, T. Sarna and J.R. Norris, J. Phys. Chem. 113 (2009), 10480-10482.

[24] S.K. Wong, D.A. Hutchinson and J.K.S. Wan, J. Chem. Phys. 58 (1973), 985-989. 


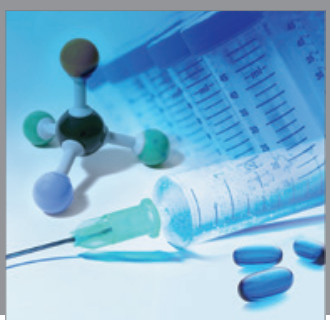

International Journal of

Medicinal Chemistry

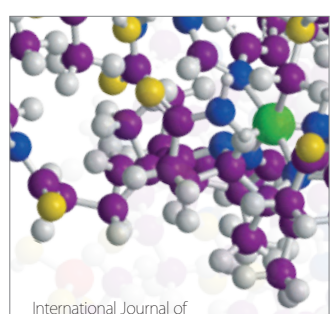

Carbohydrate Chemistry

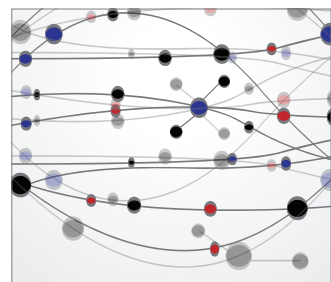

The Scientific World Journal
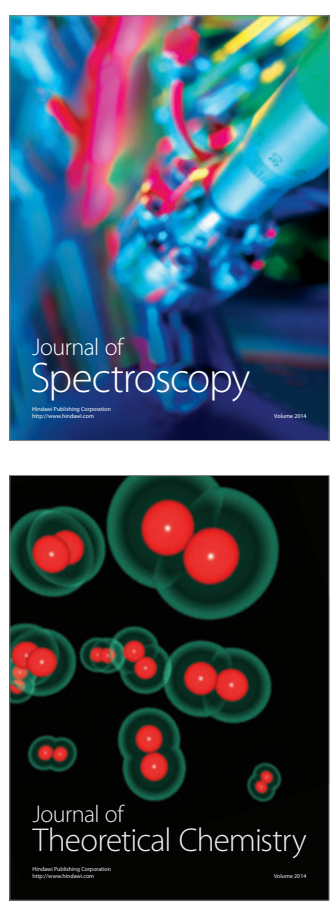
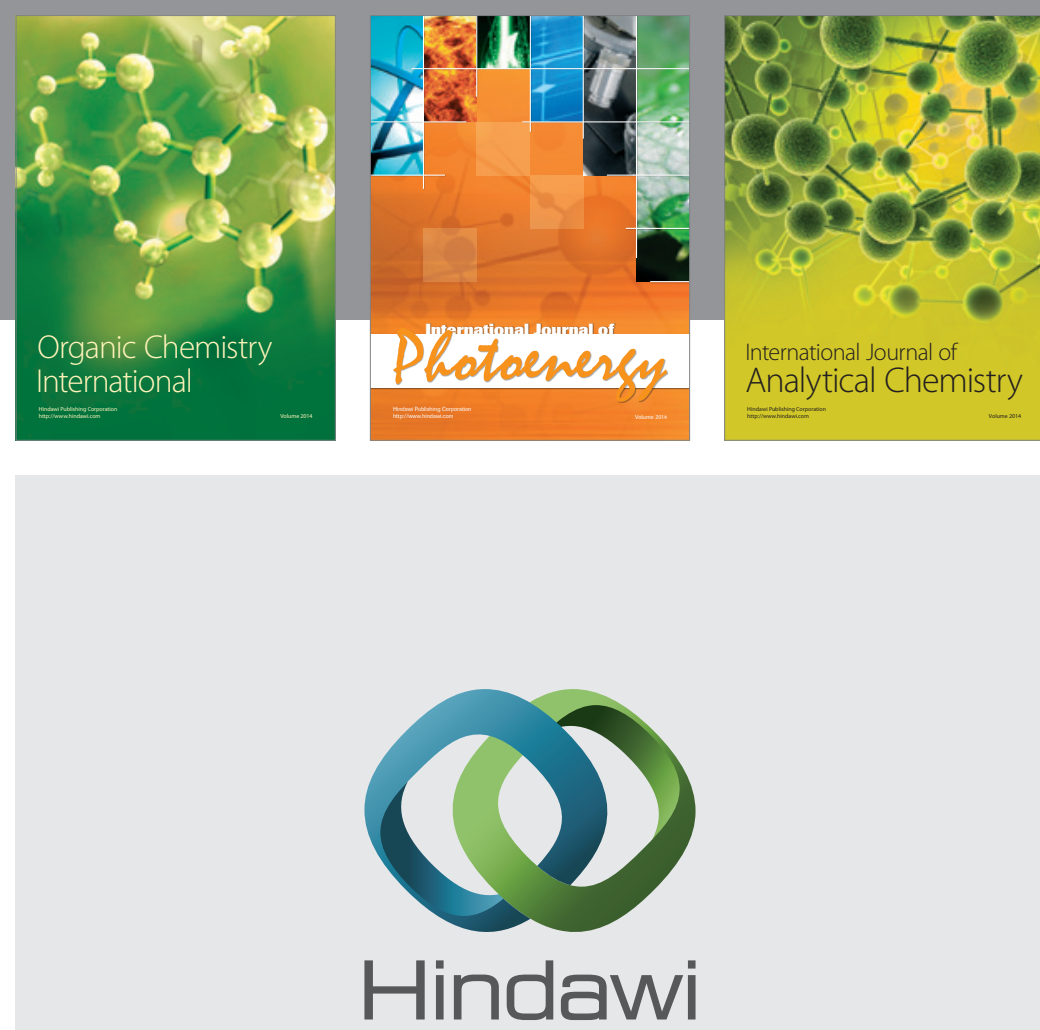

Submit your manuscripts at

http://www.hindawi.com
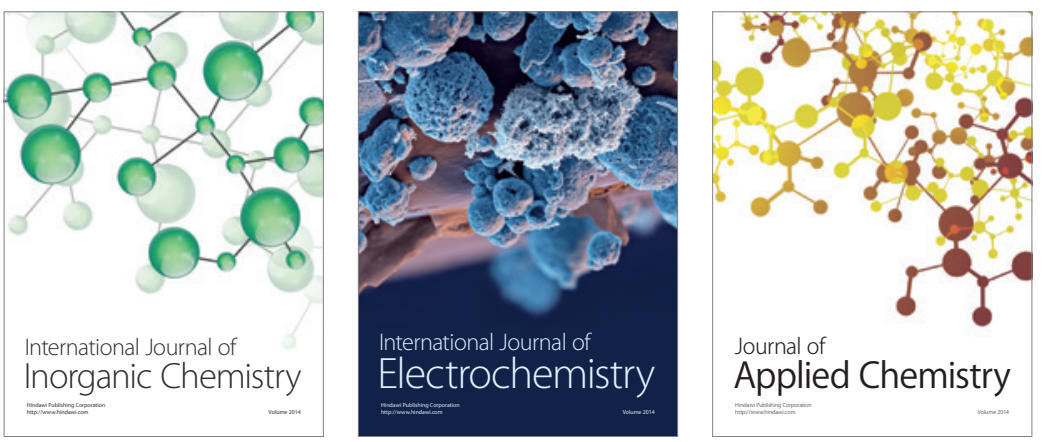

Journal of

Applied Chemistry
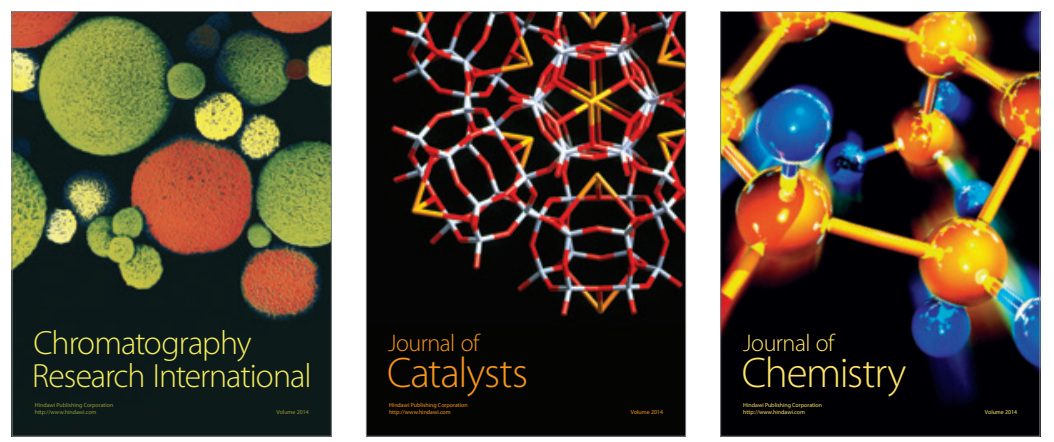
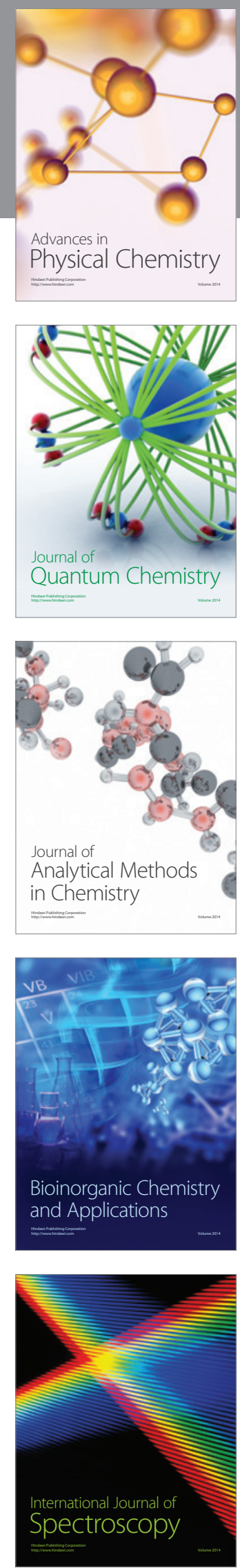